\title{
Sieve Bootstrap Prediction Intervals for Contaminated Non-linear Processes
}

\author{
Gustavo Ulloa ${ }^{1}$, Héctor Allende-Cid ${ }^{1}$, and Héctor Allende ${ }^{1,2}$ \\ 1 Universidad Técnica Federico Santa María, \\ Dept. de Informática, Casilla 110-V, Valparaíso, Chile \\ gulloa@alumnos.inf.utfsm.cl, \{vector, hallende\}@inf.utfsm.cl \\ 2 Universidad Adolfo Ibañez, Facultad de Ingeniería y Ciencias, \\ Avenida Padre Hurtado 750, Viña del Mar, Chile \\ hallende@uai.cl
}

\begin{abstract}
Recently, the sieve bootstrap method has been successfully used in prediction of nonlinear time series. In this work we study the performance of the prediction intervals based on the sieve bootstrap technique, which does not require the distributional assumption of normality as most techniques that are found in the literature. The construction of prediction intervals in the presence of patchy outliers are not robust from a distributional point of view, leading to an undesirable increase in the length of the prediction intervals.

In the analysis of financial time series it is common to have irregular observations that have different types of outliers, isolated and in groups. For this reason we propose the construction of prediction intervals for returns based in the winsorized residual and bootstrap techniques for financial time series. We propose a novel, simple, efficient and distribution free resampling technique for developing robust prediction intervals for returns and volatilities for TGARCH models. The proposed procedure is illustrated by an application to known synthetic time series.
\end{abstract}

Keywords: Sieve bootstrap, Time series, Financial prediction intervals, Forecasting in time series, Winsorized filter, GARCH, TGARCH models, Volatility.

\section{Introduction}

The construction of prediction intervals in time series models with a finite number of parameters and with known innovative processes, have been widely discussed in the literature and it is known that these intervals are extremely sensitive to the presence of innovation outliers [16].

Moreover, in the last two decades several free distribution models have been proposed as an alternative for the construction of prediction intervals [15]. This alternative consists in using a resampling technique commonly known as bootstrap. Given that the realization of a time series does not satisfy the assumption of being a succession of independent and identically distributed random variables, and given the highly dependent structure which characterizes the data, 
the authors [4138] propose a bootstrap procedure based on the resampling of the residuals, which provides good results in the context of time series in comparison with other alternatives. The first proposals based on bootstrap were applied to linear $A R M A(p, q)$ models, and then extended to other nonlinear models, like $G A R C H(p, q)$, were the prediction intervals, were not only focused in future observations of the series, in this case returns, but also in the volatility.

For linear models, the sieve bootstrap approach proposed by [1] is widely used in non-parametric time series models, because of its effectiveness, computational efficiency and for being constraint-free [13/8,9,311. The idea of the Sieve bootstrap method is to approximate the observed process by a high-order autorregresive linear model, $\operatorname{AR}(\infty)$, and generate new realizations from the resampling of the residuals 3 . The first application of bootstrap to nonlinear ARCH/GARCH models was proposed by Miguel and Olave 10. The proposal consisted in the construction of prediction intervals of returns and volatilities by directly adding resampled residuals from an ARCH model to the respective point forecasts. In 2005 an extension of this proposal was presented, by taking into account the variability of the estimation of the parameters of the ARCH model, by adding an additional step, that consisted in re-estimating the ARCH parameters for each bootstrap realization of the returns [14. Pascual et al. (2006) [12] combined and extended these procedures by developing prediction intervals for both returns and volatilities from GARCH models, and the obtained prediction intervals were found to be well-calibrated, i.e., the number of observed data falling within a prediction interval coincides with the declared coverage. In 2011 Chen et. al. proposed an alternative bootstrap method that was less demanding in terms of computational cost [5]. The proposal was based in a Sieve bootstrap procedure used in linear models, because $\mathrm{ARCH} / \mathrm{GARCH}$ models can handle the AR/ARMA Box-Jenkins family of linear models representation. The linear representation is possible because the square of the $\mathrm{ARCH} / \mathrm{GARCH}$ returns is a linear process represented by AR/ARMA models [17/7. However, if the original data has innovative outliers, the classic and free distribution approaches that include the Sieve bootstrap procedure are affected by them, producing inflations in the length of the prediction interval, which is unwanted. In the present work we propose a methodology based on a winsorized filter for the residuals to mitigate the inflation in the length of the prediction interval of Sieve bootstrap algorithms proposed in 2011 [5] for ARCH/GARCH non-linear models. We show a comparative study between the prediction interval length and its coverage with respect to other proposals in the literature. The study was performed over the prediction intervals of future values of returns and volatility. The paper is presented as follows: In the next section we present the methodology used in this work. In section 3 we show some simulation results. In the last section we present some concluding remarks.

\section{Methodology}

The proposed method is an extension of the Sieve bootstrap method for $\mathrm{ARCH} / \mathrm{GARCH}$ models proposed in 2011 [5]. It consists in adding a stage in the 
algorithm, specifically step 4, which consists in computing the winsorized filter for the estimated residuals. The algorithm is described below.

\subsection{Winsorized Sieve Bootstrap Procedure of $A R C H / G A R C H$ Process}

The $A R C H$ processes (Autoregressive Conditional Heteroskedastic) introduced by Engle in 1982 [6], allow to model the variance or volatility dynamic as a function of past observations of a return series. In 1986 [2] Bollerslev proposed an extension of the ARCH models, incorporating available information on previous volatilities in the Generalized ARCH or GARCH models.

$\operatorname{GARCH}(\mathrm{p}, \mathrm{q})$ model [2]:

$$
\begin{aligned}
y_{t} & =\sigma_{t} \epsilon_{t} \\
\sigma_{t}^{2} & =\alpha_{0}+\sum_{i=1}^{p} \alpha_{i} y_{t-i}^{2}+\sum_{j=1}^{q} \beta_{j} \sigma_{t-j}^{2}
\end{aligned}
$$

where there is a restriction over its parameters $\alpha_{0} \geq 0, \alpha_{i} \geq 0$ and $\beta_{j} \geq 0$, for $i=$ $1, \ldots, p$ and $j=1, \ldots q$, with a gaussian white noise process $\epsilon_{t} \sim$ i.i.d. $(0,1)$. For this process to be weakly stationary $\sum_{i=1}^{\max (p, q)}\left(\alpha_{i}+\beta_{j}\right)<1$ must hold, implying that $\operatorname{Var}\left[y_{t}\right] \geq 0$ is satisfied.

As mentioned in section 1 the use of Sieve bootstrap in ARCH/GARCH models is possible, because the square of the returns can be represented as linear AR/ARMA models

$$
y_{t}^{2}=\alpha_{0}+\sum_{i=1}^{m}\left(\alpha_{i}+\beta_{i}\right) y_{t-i}^{2}+\nu_{t}-\sum_{j=1}^{q} \beta_{j} \nu_{t-j}
$$

where $\left\{\nu_{t}\right\}_{t=1}^{T}$ is the innovation process of $y_{t}^{2}$ and $m=\max (p, q)$.

The algorithm with the winsorized step is described below

\section{Winsorized Sieve Bootstrap Prediction Intervals for ARCH/GARCH (WSB)}

1. Estimate the ARMA coefficients $\hat{\alpha}_{0},\left(\widehat{\alpha_{1}+\beta_{1}}\right), \ldots,\left(\widehat{\alpha_{m}+\beta_{m}}\right), \hat{\beta}_{1}, \ldots, \hat{\beta}_{q}$, by means of Least Squares. Then estimate $\hat{\alpha}_{i}=\left(\widehat{\alpha_{1}+\beta_{1}}\right)-\hat{\beta}_{i}$ for $i=$ $1, \ldots, p$.

2. Estimate the residuals $\left\{\hat{\nu}_{t}\right\}_{t=m+1}^{T}$ by means of

$$
\hat{\nu}_{t}=y_{t}^{2}-\hat{\alpha}_{0}-\sum_{i=1}^{m}\left(\widehat{\left(\alpha_{i}+\beta_{i}\right.}\right) y_{t-i}^{2}+\sum_{j=1}^{q} \hat{\beta}_{j} \nu_{t-j} \text { for } t=m+1, \cdots, T
$$


3. Center the estimated residuals by means of

$$
\tilde{\nu}_{t}=\left(\hat{\nu}_{t}-\frac{1}{T-m} \sum_{t=m+1}^{T} \hat{\nu}_{t}\right)
$$

where the empirical distribution is

$$
\hat{F}_{\nu, T}(y)=\sum_{m+1}^{T} 1_{\left\{\tilde{\nu}_{t} \leq y\right\}}
$$

4. Apply the winsorized filters of order $k$ to the residuals

$$
\tilde{\nu}_{(t)}=\left\{\begin{aligned}
\tilde{\nu}_{(p+k+1)} & \text { if } t<p+k+1 \\
\tilde{\nu}_{(t)} & \text { if } p+k+1 \leq t \leq T-k \\
\tilde{\nu}_{(T-k)} & \text { if } t>T-k
\end{aligned}\right.
$$

5. Generate a resample $\left\{\nu_{t}^{*}\right\}_{t=1}^{T}$ from $\hat{F}_{\nu, T}(y)$.

6. Generate a Bootstrap sample of the squared returns $\left\{y_{t}^{2 *}\right\}_{t=1}^{T}$ with

$$
y_{t}^{2 *}=\hat{\alpha}_{0}+\sum_{i=1}^{m}\left(\widehat{\alpha_{i}+\beta_{i}}\right) y_{t-i}^{2 *}+\nu_{t}^{*}-\sum_{j=1}^{q} \hat{\beta}_{j} \nu_{t-j}^{*}
$$

where $y_{k}^{2 *}=\frac{\hat{\alpha}_{0}}{1-\sum_{i=1}^{m}\left(\widehat{\alpha_{i}+\beta_{i}}\right)}$ and $\nu_{k}^{*}=0$ for $k \leq 0$

7. Given $\left\{y_{t}^{2 *}\right\}_{t=1}^{T}$ from step 6 , estimate the coefficients $\hat{\alpha}_{0}^{*},\left(\widehat{\alpha_{1}+\beta_{1}}\right)^{*}, \ldots$, $\left(\widehat{\alpha}_{m+\beta_{m}}\right)^{*}, \hat{\beta}_{1}^{*}, \ldots, \hat{\beta}_{q}^{*}, \hat{\alpha}_{i}^{*}=\left(\widehat{\alpha_{1}+\beta_{1}}\right)^{*}-\hat{\beta}_{i}^{*}$ for $i=1, \ldots, p$. The Bootstrap sample of the volatility $\left\{\sigma_{t}^{2 *}\right\}_{t=1}^{T}$ is obtained with

$$
\sigma_{t}^{2 *}=\hat{\alpha}_{0}^{*}+\sum_{i=1}^{p} \hat{\alpha}_{i}^{*} y_{t-i}^{2 *}+\sum_{j=1}^{q} \hat{\beta}_{j}^{*} \sigma_{t-j}^{2 *} \text { for } t=m+1, \cdots, T
$$

with $\sigma_{t}^{2 *}=\frac{\hat{\alpha}_{0}}{1-\sum_{i=1}^{m}\left(\hat{\alpha}_{i}+\hat{\beta}_{i}\right)}$, for $t=1, \cdots, m$.

8. Sample with replacement from $\hat{F}_{\nu, T}(y)$ to obtain the Bootstrap prediction error process $\left\{\nu_{t+h}^{*}\right\}_{h=1}^{s}$ where $s \geq 1$.

9. $\quad$ Be $y_{T+h}^{*}=y_{T+h}, \nu_{T+h}^{*}=\tilde{\nu}_{T+h} \mathrm{y} \sigma_{T+h}^{2 *}=\sigma_{T+h}^{2 *}$ for $h \leq 0$

$$
\begin{gathered}
y_{T+h}^{2 *}=\hat{\alpha}_{0}^{*}+\sum_{i=1}^{m}\left(\widehat{\alpha_{i}+\beta_{i}}\right)^{*} y_{T+h-i}^{2 *}+\nu_{T+h}^{*}-\sum_{j=1}^{q} \hat{\beta}_{j}^{*} \nu_{T+h-j}^{*} \\
\sigma_{T+h}^{2 *}=\hat{\alpha}_{0}^{*}+\sum_{i=1}^{p} \hat{\alpha}_{i}^{*} y_{T+h-i}^{2 *}+\sum_{j=1}^{q} \hat{\beta}_{j}^{*} \sigma_{T+h-j}^{2 *} \text { for } h=1, \ldots, s
\end{gathered}
$$

10. Repeat steps 4 to $8, B$ times. 
11. Finally, prediction intervals are obtained $(1-\alpha) \%$ for $y_{T+h}$ y $\sigma_{T+h}^{2}$ using $\hat{F}_{y_{T+h}^{2 *}}^{*}$ and $\hat{F}_{\sigma_{T+h}^{2 *}}^{*}$.

- For $y_{T+h}$ :

$$
\left[-\sqrt{H_{(1-\alpha)}^{*}}, \sqrt{H_{(1-\alpha)}^{*}}\right], h=1, \cdots, s
$$

where $H_{(1-\alpha)}^{*}$ is the quantile $1-\alpha$ of $\hat{F}_{y_{T+h}^{2 *}}^{*}$

- For $\sigma_{T+h}^{2}$ :

$$
\left[0, K_{(1-\alpha)}^{*}\right], h=1, \cdots, s
$$

where $K_{(1-\alpha)}^{*}$ is the quantile $1-\alpha$ of $\hat{F}_{\sigma_{T+h}^{2 *}}^{2 *}$

\section{Simulation Results}

In this section we compared our proposal (WSB) with state of the art models applied to nonlinear time series models (GARCH).

The following tables show the results of the comparison of the models regarding coverage and length of the intervals, in addition of the combined metric CQM proposed in [1], which depends of the theoretical coverage and length, and the empirical values obtained with the bootstrap prediction intervals. This metric is a discrepancy metric between the theorical aim and the empirical performance, for that, a smaller value obtained in this metric indicates a better performance of the prediction interval.

\subsection{Simulation for Nonlinear ARCH/GARCH Models}

We compared the proposed method (WSB) with the proposal [5] for prediction intervals of future values and volatility.

The parameters of the simulation were: $B=1000$ resamples of $X_{T+h}^{*}$ and $\sigma_{T+h}^{2 *}, R=1000$ future values $X_{T+h}$ and $\sigma_{T+h}^{2}$ were simulated for each $h=1,5$ and 10 step-ahead. $S=1000$ simulated the $A R C H(2)$ process

$$
\begin{gathered}
y_{t}=\sigma_{t} \cdot \varepsilon_{t} \\
\sigma_{t}^{2}=0.1+0.2 y_{t-1}^{2}+0.15 y_{t-2}^{2}
\end{gathered}
$$

with the two different distributions for the innovation process $\left\{\varepsilon_{t}\right\}_{t \in \mathbb{Z}}$ given by

$$
F_{\varepsilon_{t}}=N(0,1)
$$

and

$$
F_{\varepsilon_{t}}=(1-\zeta) N(0,1)+\zeta N(0,100)
$$

where (17) is the contaminated innovations process generated with the convex linear distribution

$$
F_{\varepsilon_{t}}=(1-\zeta) N(0,1)+\zeta N\left(0, \sigma^{2}\right)
$$

where $\sigma^{2}=100$ with a $\zeta=0.05$ level of contamination. 
For each simulated series the same procedure described in subsection 2.1 was performed.

In tables 1 and 2 we present the results of the comparison of the prediction interval for $h=1,5$ and 10 steps-ahead of the two methods with respect to their average coverage, length of the intervals and the combined measure CQM.

In Table 1 we observe the results of the simulation of the innovative process without contamination (16). We observe that algorithm (SB) and the proposed (WSB) with different orders $k$, have similar results in terms of coverage and empirical length, and that taking the residuals out of the extremes, decreases a bit the performance, because the CQM value increases its magnitude. The 1-step ahead predictions of volatility given by (11) are constant, so the CQM value can not be calculated.

It is observed that under the presence of contamination in the innovative process the prediction intervals of the algorithm (SB) are clearly affected by the coverture of the returns and volatility, and also the increment in the length of them. This can be seen in Table 2 by comparing the coverture and length of the prediction intervals of the proposed method (SB) with the empirical coverture and length. Also it is observed that the method (WSB) has a positive impact in the performance of the prediction intervals of the returns and volatility. As the order $k$ of the proposed method is increased, the magnitude of the measure CQM decreases for the returns and the volatility, thus diminishing the inflation of the prediction intervals and average covertures. It seems that if the filter order of algorithm WSB increases, the covertures and lengths of the prediction intervals converge to the theoretical covertures and lengths.

Table 1. Results of the simulation of model $\mathrm{ARCH}(2)$ with an innovation process $N(0,1)$

\begin{tabular}{|c|c|c|c|c|c|c|c|c|}
\hline $\mathrm{h}$ & $\mathrm{k}$ & Method & $\begin{array}{c}\text { Coverture } \\
\text { return (d.e.) }\end{array}$ & $\begin{array}{c}\text { Length } \\
\text { return (d.e.) }\end{array}$ & $\begin{array}{c}\text { CQM } \\
\text { return }\end{array}$ & $\begin{array}{c}\text { Coverture } \\
\text { volatility (d.e.) }\end{array}$ & $\begin{array}{c}\text { Length } \\
\text { volatility (d.e.) }\end{array}$ & $\begin{array}{c}\text { CQM } \\
\text { volatility }\end{array}$ \\
\hline $\mathrm{h}=1$ & - & EMP & $95 \%$ & 1.52 & - & $95 \%$ & 0.00 & - \\
& - & SB & $94.71(3.23)$ & $1.52(0.12)$ & 0.005 & $88.9(31.41)$ & $0.17(0.08)$ & - \\
& 1 & WSB & $94.70(3.23)$ & $1.52(0.12)$ & 0.005 & $88.00(32.50)$ & $0.17(0.08)$ & - \\
& 2 & WSB & $94.69(3.24)$ & $1.52(0.12)$ & 0.006 & $87.90(32.61)$ & $0.17(0.08)$ & - \\
& 3 & WSB & $94.69(3.24)$ & $1.52(0.12)$ & 0.006 & $86.90(33.74)$ & $0.17(0.08)$ & - \\
\hline $\mathrm{h}=5$ & - & EMP & $95 \%$ & 1.55 & - & $95 \%$ & 0.23 & - \\
& - & SB & $94.77(1.37)$ & $1.54(0.08)$ & 0.008 & $93.77(3.72)$ & $0.27(0.05)$ & 0.211 \\
& 1 & WSB & $94.72(1.37)$ & $1.54(0.08)$ & 0.008 & $93.70(3.73)$ & $0.27(0.05)$ & 0.207 \\
& 2 & WSB & $94.70(1.37)$ & $1.53(0.08)$ & 0.012 & $93.63(3.79)$ & $0.27(0.05)$ & 0.204 \\
& 3 & WSB & $94.67(1.37)$ & $1.53(0.08)$ & 0.014 & $93.53(3.81)$ & $0.27(0.05)$ & 0.200 \\
\hline $\mathrm{h}=10$ & - & EMP & $95 \%$ & 1.55 & - & $95 \%$ & 0.23 & - \\
& - & SB & $94.74(1.33)$ & $1.54(0.08)$ & 0.009 & $93.74(3.72)$ & $0.27(0.05)$ & 0.214 \\
& 1 & WSB & $94.71(1.34)$ & $1.53(0.08)$ & 0.011 & $93.67(3.75)$ & $0.27(0.05)$ & 0.210 \\
& 2 & WSB & $94.67(1.33)$ & $1.53(0.08)$ & 0.013 & $93.60(3.78)$ & $0.27(0.05)$ & 0.206 \\
& 3 & WSB & $94.67(1.33)$ & $1.53(0.08)$ & 0.015 & $93.53(3.80)$ & $0.27(0.05)$ & 0.202 \\
\hline
\end{tabular}


Table 2. Results of the simulation of model $\mathrm{ARCH}(2)$ with an innovation process $F_{\varepsilon_{t}}=(1-\zeta) N(0,1)+\zeta N(0,100)$

\begin{tabular}{|c|c|c|c|c|c|c|c|c|}
\hline $\mathrm{h}$ & $\mathrm{k}$ & Method & $\begin{array}{c}\text { Coverture } \\
\text { return (d.e.) }\end{array}$ & $\begin{array}{c}\text { Length } \\
\text { return (d.e.) }\end{array}$ & $\begin{array}{c}\text { CQM } \\
\text { return }\end{array}$ & $\begin{array}{c}\text { Coverture } \\
\text { volatility (d.e.) }\end{array}$ & $\begin{array}{c}\text { Length } \\
\text { volatility (d.e.) }\end{array}$ & $\begin{array}{c}\text { CQM } \\
\text { volatility }\end{array}$ \\
\hline $\mathrm{h}=1$ & - & EMP & $95 \%$ & 2.44 & - & $95 \%$ & - & - \\
& - & SB & $98.34(6.11)$ & $7.86(15.71)$ & 2.248 & $98.40(12.55)$ & $112.43(1236.36)$ & - \\
& 1 & WSB & $97.33(8.24)$ & $4.57(3.59)$ & 0.898 & $97.90(14.34)$ & $14.85(81.14)$ & - \\
& 2 & WSB & $96.52(9.76)$ & $2.94(2.59)$ & 0.647 & $97.80(14.67)$ & $7.74(34.23)$ & - \\
& 3 & WSB & $96.26(9.78)$ & $3.71(2.04)$ & 0.544 & $97.70(14.99)$ & $4.99(15.92)$ & - \\
\hline $\mathrm{h}=5$ & - & EMP & $95 \%$ & 1.78 & - & $95 \%$ & 0.94 & - \\
& - & SB & $99.73(0.84)$ & $10.55(18.51)$ & 4.969 & $99.74(1.31)$ & $144.36(1537.29)$ & 152.633 \\
& 1 & WSB & $99.51(2.51)$ & $6.99(4.95)$ & 2.970 & $99.62(1.97)$ & $20.85(107.61)$ & 21.186 \\
& 2 & WSB & $99.52(1.83)$ & $6.35(3.71)$ & 2.616 & $99.57(2.16)$ & $11.44(56.02)$ & 11.292 \\
& 3 & WSB & $99.48(2.10)$ & $5.97(2.65)$ & 2.397 & $99.52(2.34)$ & $7.28(16.23)$ & 6.792 \\
\hline $\mathrm{h}=10$ & - & EMP & $95 \%$ & 1.61 & - & $95 \%$ & 0.29 & - \\
& - & SB & $99.83(0.18)$ & $10.89(18.87)$ & 5.803 & $99.89(0.17)$ & $149.95(1579.38)$ & 507.893 \\
& 1 & WSB & $99.77(0.34)$ & $7.38(5.49)$ & 3.627 & $99.84(0.21)$ & $23.06(113.41)$ & 77.270 \\
& 2 & WSB & $99.55(3.24)$ & $6.67(4.36)$ & 3.187 & $99.82(0.23)$ & $13.56(67.12)$ & 45.054 \\
& 3 & WSB & $99.51(3.03)$ & $6.25(3.35)$ & 2.92 & $99.79(0.25)$ & $8.79(19.69)$ & 28.800 \\
\hline
\end{tabular}

\section{Conclusion}

In 1] and [5] the authors show that the Sieve bootstrap procedures obtains better results than state of the art models for coverage and length of prediction intervals and combined metric CQM.

In this work we show the effects of the presence of innovation outliers in prediction intervals, which extend their length and coverage. We also show how to mitigate these effects by applying a winsorized filter to the residual estimates.

The proposed method generates prediction intervals that are more accurate in coverage terms and with shorter length. These improvements are reflected in a lower value of the CQM metric.

As future work we propose the search for a method to identify the optimal order $k$ of the filter and we plan to test our proposal with real nonlinear time series with outliers in order to perform a comparative study.

Acknowledgment. This work was supported by the following Research Grants: Fondecyt 1110854 and FB0821 Centro Científico Tecnológico de Valparaíso. Partial support was also received from Mecesup FSM 0707.

\section{References}

1. Alonso, A., Peña, D., Romo, J.: Forecasting time series with sieve bootstrap. Journal of Statistical Planning and Inference 100, 1-11 (2002)

2. Bollerslev, T.: Generalized autorregressive conditional heteroskedasticity. Journal of Econometrics 31, 307-327 (1986)

3. Bühlmann, P.: Sieve bootstrap for time series. Bernoulli 2(3), 123-148 (1997) 
4. Bühlmann, P.: Bootstrap for time series. Statistical Science 17(1), 52-72 (2002)

5. Chen, B., Gel, Y., Balakrishna, N., Abraham, B.: Computationally efficient bootstrap prediction intervals for returns and volatilities in arch and garch processes. Journal of Forecasting 30, 51-71 (2011)

6. Engle, R.F.: Autorregressive conditional heteroskedasticity with estimates of the variance of united kindom inflation. Econometrica 50, 987-1007 (1982)

7. Francq, C., Zakoian, J.: GARCH Models: Structure, statistical inference and financial applications. Wiley (2010)

8. Härdle, W.: Bootstrap methods for time series. International Statistical Review 71, 435-459 (2003)

9. Kreiss, J.P.: Asymptotic statistical inference for a class of stochastic processes. Habilitationsschrift, Universität Hamburg (1998)

10. Miguel, J., Olave, P.: Bootstrapping forecast intervals in arch models. Sociedad de Estadıstica e Investigación Operativa 8(2), 345-364 (1999)

11. Pascual, L., Romo, J., Ruiz, E.: Bootstrap predictive inference for arima processes. Journal of Time Series Analysis 25, 449-465 (2004)

12. Pascual, L., Romo, J., Ruiz, E.: Bootstrap prediction for returns and volatilities in garch models. Computational Statistics and Data Analysis 50, 2293-2312 (2006)

13. Politis, D.N.: The impact of bootstrap methods on time series analysis. Statistical Science 28, 219-230 (2003)

14. Reeves, J.J.: Bootstrap prediction intervals for arch models. Internal Journal of Forecasting 21, 237-248 (2005)

15. Thombs, L.A., Schucany, W.R.: Bootstrap prediction intervals for autoregression. Journal of the American Statistical Association 85, 486-492 (1990)

16. Tsay, R.S.: Outliers, level shifts, and variance changes in time series. J. Forecast 7, 1-20 (1988)

17. Tsay, R.S.: Analysis of Financial Time Series, 2nd edn. Wiley Interscience (2005) 